\title{
Semiclassical collision theory. Multidimensional Bessel uniform approximation *
}

\author{
H. Kreek, R. L. Ellis, and R. A. Marcus \\ Department of Chemistry, University of Illinois, Urbana, Illinois 61801 \\ (Received 13 June 1974)

\begin{abstract}
A multidimensional Bessel uniform approximation for the semiclassical $\mathbf{S}$ matrix is derived for the case of four real stationary phase points. A formula is also developed for the particular case when four stationary phase points may be considered to be well separated in pairs. The latter equation is then used in the treatment of two real and two complex stationary phase points.
\end{abstract}

\section{INTRODUCTION}

The following integ ral arises in the semiclassical treatment $t^{1-5}$ of inelastic collisions:

$$
I=(2 \pi)^{-r} \int_{-\pi}^{\pi} \ldots \int_{-\pi}^{\pi} g(\mathbf{x}) \exp [i f(\mathbf{x})] d x_{1} \ldots d x_{r} .
$$

Here, $\mathbf{x}\left(x_{1} \ldots, x_{r}\right)$ denotes the certain "reduced" phases (related to coordinates in the "action-angle" variables) of the colliding species.

When the integral in Eq. (1.1) is evaluated by a simple stationary phase method one obtains the primitive semiclassical (PSC) expression for the value of $I$. When the integral is evaluated by mapping the exponent $f(x)$ onto a cubic function, ${ }^{1 \mathrm{c}}$ and the integration limits of $(-\pi, \pi)$ are replaced by $(-\infty, \infty)$, an Airy uniform approximation of $I$ is obtained. Quantum mechanically it is more accurate than the PSC value. However, when $f(\mathbf{x})$ varies very slowly with $\mathbf{x}$, large portions of the $\mathbf{x}$ domain contribute to the integral, and approximations such as replacing the interval $(-\pi, \pi)$ by $(-\infty, \infty)$ break down, and with it, the Airy uniform approximation. ${ }^{11}$

For this reason Stine and one of us introduced a more accurate mapping which preserves the desired periodicity properties of the $(-\pi, \pi)$ interval ${ }^{11}: f(\mathbf{x})$ was mapped onto a sinusoidal plus linear function, yielding a Bessel uniform approximation. Unlike the Airy the Bessel uniform approximation does not break down when $f(\mathbf{x})$ is slowly varying, ${ }^{11}$ and so is more general and includes the Airy as a special case. To be sure, it, too, is expected to have its limitations for sufficiently unusual $f(x)$ and $g(\mathbf{x})$ in Eq. (1.1).

An Airy uniform approximation to Eq. (1.1) has been given for $r$ equal to one, ${ }^{1 \mathrm{c}, 2 \mathrm{~b}}$ two, ${ }^{1 \mathrm{~h}, 2 \mathrm{c}, 3}$ and more than two, ${ }^{1 \mathrm{~h}, 3}$ while the Bessel function uniform approximation has been given for $r$ equal to one. ${ }^{11}$ In the present paper, a Bessel uniform approximation is developed for $r \geq 2$. Initially, the prototype, a two-dimensional integral (1.2) is treated. The argument is then generalized to higher dimensions:

$$
I=(2 \pi)^{-2} \int_{-\pi}^{r} \int_{-\Gamma}^{r} g(x, y) \exp [i f(x, y)] d x d y .
$$

Perhaps it would be useful to note first that the integral (1.1) can be reduced in some applications to a onedimensional integral (then circumventing the present derivation), but in other cases, it cannot: The integral $I$ yields the probability amplitude for a transition from some initial quantum state of a colliding pair of molecules, defined by quantum numbers $\left(n_{1}, \ldots, n_{r}\right)$, to some final state $\left(m_{1}, \ldots, m_{r}\right)$. If one is interested only in the transition of $n_{1}$, say, i.e., in $n_{1}-m_{1}$, one can first average the transition probability over the ensemble distribution of $\left(n_{2}, \ldots, n_{r}\right)$ appropriate to the experiment. If, further, many final quantum states $\left(m_{2}, \ldots, m_{r}\right)$ are accessible for each of the $m_{i}$ 's, one can integrate over $\left(m_{2}, \ldots, m_{r}\right)$ using "partial averaging." $2 \mathrm{~h}$ A one-dimensional integral remains and is treated by available (stationary phase, integral or uniform) methods. When instead, only a few final quantum states for some of the $m_{2}, \ldots, m_{r}$ degrees of freedom are accessible, or when one is measuring a particular simultaneous transition $\left(n_{1}, \ldots, n_{s}\right) \rightarrow\left(m_{1}, \ldots, m_{s}\right)$, with $1<s \leqslant r$, a two or more dimensional integral over $d x_{1} \ldots d x_{s}$ remains and the present formulation of a multidimensional Bessel uniform approximation or Bessel uniform-in-pairs approximation can be applied.

\section{BESSEL UNIFORM APPROXIMATION}

In developing a Bessel uniform approximation to the integral given in $\mathrm{Eq}$. (1.2), we shall use a procedure analogous to that developed in a previous paper in this series. $^{1 h}$ First, a one-to-one mapping $(x, y) \rightarrow(u, v)$ is performed, to convert $f(x, y)$ to the form of Eqs. (2.1) and (2.2). The function $f(x, y)$ in Eq. (1.2) is mapped onto a family of curves in the $u$ direction by ${ }^{6}$

$$
f(x, y)=K u-\zeta \sin u+A \equiv \bar{f}(u, v) .
$$

This family of curves are referred to as $u$-curves, and a few members of the family are shown schematically in Fig. $1{ }^{7}$ In Eq. (2.1) $K$ is an integer, dictated by the physical problem, and is the same for all members of the family of $u$ curves. $\zeta$ and $A$ are constant along any member of the family, but may differ from member to member. These "constants" thus can depend on both $u$ and $v$.

Similarly, curves designated as $v$ curves in Fig. 1 are chosen such that

$$
f(x, y)=k v-\zeta \sin v+A \equiv \bar{f}(u, v),
$$

where $k$ is an integer constant dependent on the physical problem and $\zeta$ and $A$ are constants on a particular $v$ curve, and dependent on both $u$ and $v$. The $A$ and $\zeta$ in Eq. (2.2) differ from the ones in Eq. (2.1).

The $\zeta$ and $A$ in Eqs. (2.1) and (2.2) are related to the properties of the extrema of $\bar{f}$ on the $u$ and $v$ curves as follows: These extrema are defined as the points at 


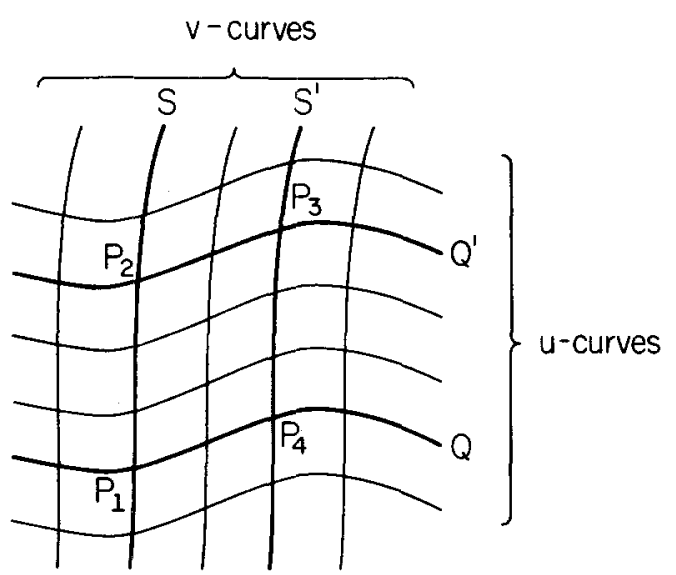

FIG. 1. A schematic representation of $u$ curves and $v$ curves. The stationary phase points $P_{1}$ and $P_{3}$ are the maximum and minimum respectively, and $P_{2}$ and $P_{4}$ are saddle points. The curves $S$ and $S^{1}$ are respectively the locus of maximum and minimum on $u$ curves. The curves $Q$ and $Q^{1}$ are respectively the locus of maximum and minimum on $v$ curves.

which $d \bar{f} / d u(d \bar{f} / d v)$ vanish along a $u$ curve ( $v$ curve). On any $u$ curve these points are determined from $\mathrm{Eq}$. (2. 1) by

$$
K-\varepsilon \cos u=0
$$

and on any $v$ curve by

$$
k-\xi \cos v=0 \text {. }
$$

Of the infinite number of roots of Eqs. (2.3a) and (2.3b) we shall select those, denoted by $u_{s}, u_{s^{\prime}}$ and $v_{Q}, v_{Q^{\prime}}$, which lie in the interval $(-\pi, \pi)$. They have the property that

$$
u_{S}=-u_{S^{\prime}} ; \quad v_{Q}=-v_{0^{\prime}} .
$$

We can further classify these roots by the behavior of $d^{2} \bar{f} / d u^{2}$ and $d^{2} \bar{f} / d v^{2}$ on their respective curves. From Eqs. (2.3a) and (2.3b)

$$
d^{2} \bar{f} / d u^{2}=\zeta \sin u
$$

and

$$
d^{2} \bar{f} / d v^{2}=\zeta \sin v
$$

We shall distinguish $u_{S}$ from $u_{S}$, and $v_{Q}$ from $v_{Q}$, by the conditions:

$$
\begin{array}{ll}
\zeta \sin u_{S}<0, & \zeta \sin u_{S^{\prime}}>0 \\
\zeta \sin v_{Q}<0, & \zeta \sin v_{Q^{\prime}}>0 .
\end{array}
$$

This identification specifies that $u_{s}$ and $v_{Q}$ are maxima, and $u_{S^{\prime}}$ and $v_{Q^{\prime}}$ are minima. ${ }^{8}$ If either of the derivatives in Eq. (2.5) equals zero then the roots have coalesced to form an inflection point. Figure 1 shows schematically the curve, labeled by $S$ of all points $u_{S}$. Similarly those labeled by $S^{\prime}, Q^{\prime}$, and $Q$, from the loci of the points $u_{S^{\prime}}, v_{Q^{\prime}}$ and $v_{Q}$, respectively. The points $P_{1}, P_{2}$, $P_{3}$, and $P_{4}$ at the pairwise intersections of these four curves correspond to the four points of stationary phase of $f(x, y)$. Using the definition in Eqs. (2.5) and (2.6), it is seen that $P_{1}$ and $P_{3}$ are, respectively, a maximum and minimum, and that $P_{2}$ and $P_{4}$ are saddle points.

At any pair of points $u_{S}$ and $u_{s}$, which satisfy Eq. (2. 3a) the value of $\zeta$ and $A$ can be obtained by substitution into Eq. (2.1). Then

$$
\begin{aligned}
& A_{S^{\prime}}=\frac{1}{2}\left(f_{S^{\prime}}+f_{S}\right), \\
& K\left(u_{S^{\prime}}-\tan u_{S^{\prime}}\right)=\frac{1}{2}\left(f_{S^{\prime}}-f_{S^{\prime}}\right) .
\end{aligned}
$$

Because $f_{S^{\prime}}-f_{S}$ is negative by definition, then when $K<0$ the solution $u_{S}$, of $(2.7 \mathrm{~b})$ must lie, one can show, in the $\left(-\frac{1}{2} \pi, 0\right)$ interval. When $K>0$, it lies in the $\left(0, \frac{1}{2} \pi\right)$ interval. That is,

$$
-\frac{\mathrm{r}}{2} \pi<u_{S^{\prime}}<0 \quad(K<0), 0<u_{S^{\prime}}<\frac{1}{2} \pi \quad(K>0) .
$$

With this restriction the $\zeta$ are now determined from

$$
\zeta_{S s^{\prime}}=K / \cos u_{s^{\prime}}(K \neq 0) \text {. }
$$

When $K=0, A_{S s^{\prime}}$ is given by Eq. (2.7a), and instead of Eqs. (2.7b) and (2.8), we have

$$
\zeta_{s s^{\prime}}=\frac{1}{2}\left(f_{S^{\prime}}-f_{S}\right) ; u_{s^{\prime}}=-\frac{1}{2} \pi \quad(K=0) \text {. }
$$

In terms of $u$ and $v$ Eq. (1.2) becomes

$I=\left(4 \pi^{2}\right)^{-1} \int_{-\pi}^{\pi} \int_{-\pi}^{\pi}(g J)_{s s^{\prime}} \exp \left[i\left(K u-\zeta_{S s^{\prime}} \sin u+A_{S^{\prime}}\right)\right] d u d v$,

where $J(x, y ; u, v)$ is the Jacobian of the transformation from $x, y$ to $u, v$. The function $g J$ is different for each member $S S^{\prime}$ of the family of $u$ curves. Consequently it is subscripted $S S^{\prime}$. We assume that on any $u$ curve $S S^{\prime}$ $\left(g_{J} J\right)_{S S}$ may be written as

$$
(g J)_{S S^{\prime}}=r+t \sin u \text {. }
$$

Each $u$ curve has its characteristic value of $r$ and of $t$, and these values for a given $u$ curve are determined from the value of $g J$ at $u_{S}$ and $u_{S^{\prime}},(g J)_{S}$ and $(g J)_{S^{\prime}}$, respectively. From Eq. (2.11) one finds

$$
\begin{aligned}
& r=\frac{1}{2}\left[(g J)_{s^{\prime}}+(g J)_{s}\right] ; \\
& t=\left(2 \sin u_{s^{\prime}}\right)^{-1}\left[(g J)_{s^{\prime}}-(g J)_{s}\right] .
\end{aligned}
$$

Substituting Eqs. (2.11) and (2.12) into Eq. (2.10) yields

$$
\begin{aligned}
I=\left(8 \pi^{2}\right)^{-1} & \int_{-\pi}^{\pi} \int_{-\pi}^{\pi}\left[(g J)_{s^{\prime}}+(g J)_{s}\right] \exp \left[i\left(K u-\zeta_{s s^{\prime}} \sin u+A_{S_{s^{\prime}}}\right] d u d v+\left(8 \pi^{2}\right)^{-1}\right. \\
& \times \int_{-\pi}^{\pi} \int_{-\pi}^{\pi}\left(\sin u_{s^{\prime}}\right)^{-1}\left[(g J)_{s^{\prime}}-(g J)_{s}\right] \sin u \exp \left[i\left(K u-\zeta_{s s^{\prime}} \sin u+A_{s^{\prime}}\right)\right] d u d v
\end{aligned}
$$

The integral over $u$ is related to Bessel functions of integral order. ${ }^{9}$ To simplify the notation we define a function $\mathfrak{g}$ in terms of a Bessel function and its derivative:

$$
\mathfrak{g}_{K}^{ \pm}\left(\zeta_{S s^{\prime}}\right)=J_{K}\left(\zeta_{S^{\prime}}\right) \mp i J_{K}^{\prime}\left(\zeta_{S s^{\prime}}\right)\left(\sin u_{s^{\prime}}\right)^{-1},
$$

where

$$
\begin{aligned}
& J_{K}\left(\zeta_{s s^{\prime}}\right)=(2 \pi)^{-1} \int_{-\pi}^{\pi} \exp \left[i\left(K u-\zeta_{s s^{\prime}} \sin u\right)\right] d u, \\
& J_{K}^{\prime}\left(\zeta_{s s^{\prime}}\right)=d J_{K}\left(\zeta_{s s^{\prime}}\right) / d \zeta_{s s^{\prime}}
\end{aligned}
$$




$$
=(2 \pi i)^{-1} \int_{-\pi}^{r} \sin u \exp \left[i\left(K u-\zeta_{s s^{\prime}} \sin u\right)\right] d u .
$$

The integration over $u$ in Eq. (2.13) may not be performed, with the result

$$
\begin{aligned}
& I=(4 \pi)^{-1} \int_{-\pi}^{\pi} \exp \left(i A_{S S^{\prime}}\right) {\left[(g J)_{S^{\prime}} \mathcal{J}_{K}^{-}\left(\zeta_{S s^{\prime}}\right)\right.} \\
&\left.+(g J)_{S} \partial_{K}^{+}\left(\zeta_{S s^{\prime}}\right)\right] d v .
\end{aligned}
$$

The integration over $v$ in Eq. (2.16) could be performed in a similar manner if one could separate variables, for then $\zeta_{s s^{\prime}}$ would be independent of $v$. Instead, since typically $\zeta_{s} s^{\prime}$ is dependent on $v$, it is convenient to assume that $\zeta_{s s^{\prime}}$ is sufficiently large that $\mathcal{J}^{ \pm}\left(\zeta_{s s^{\prime}}\right)$ almost has its asymptotic form

$$
\begin{aligned}
\mathfrak{J}_{K}^{ \pm}\left(\zeta_{s s^{\prime}}\right) \cong\left[2 / \pi \zeta_{s s^{\prime}} \sin u_{s^{\prime}}\right]^{1 / 2} \\
\\
\times \exp \left[\mp i\left(K u_{s^{\prime}}-\zeta_{s s^{\prime}} \sin u_{s^{\prime}}+\frac{1}{4} \pi\right)\right]\left(\operatorname{large} \zeta_{s s^{\prime}}\right)
\end{aligned}
$$

We then introduce a new function $\varkappa_{K}^{ \pm}\left(\zeta s s^{\prime}\right)$ via Eq.

(2.18). For sufficiently large $\zeta_{s s^{\prime}} \mathcal{K}_{K}^{ \pm}\left(\zeta_{s s^{\prime}}\right)$ approaches unity, and for somewhat smaller $\zeta_{s} s^{\prime}$ it is only weakly dependent on $v$ :

$$
\begin{aligned}
\varkappa_{K}^{ \pm}\left(\zeta_{s s^{\prime}}\right)= & \left(\pi \zeta_{s s^{\prime}} \sin u_{s^{\prime}} / 2\right)^{1 / 2} g_{K}^{ \pm}\left(\zeta_{s^{\prime}}\right) \\
& \times \exp \left[ \pm i\left(K u_{s^{\prime}}-\zeta_{s s^{\prime}} \sin u_{s^{\prime}}+\frac{1}{4} \pi\right)\right]
\end{aligned}
$$

The integral in Eq. (2.16) now becomes

$$
I=(4 \pi)^{-1} \int_{-\pi}^{\pi}\left(2 / \pi \zeta_{s s^{\prime}} \cdot \sin u_{s^{\prime}}\right)^{1 / 2}\left\{\left[(g J)_{s^{\prime}} ; \mathcal{K}_{K}^{-}\left(\zeta_{s s^{\prime}}\right)\right.\right.
$$

$$
\left.\times \exp \left[i\left(f_{S^{\prime}}+\frac{1}{4} \pi\right)\right]+(g \jmath)_{S} \mathcal{K}_{K}^{+}\left(\zeta_{S S^{\prime}}\right) \exp \left[i\left(f_{S}-\frac{1}{4} \pi\right)\right]\right\} d v .
$$

We next assume that the pre-exponential factor of the terms in Eq. (2.19) have a sufficiently slowly varying phase, as a function of $v$, that they may be mapped onto a function of the form $R+T \sin v$ along the $v$ curve composed of $u_{\mathrm{S}}$, points. Along the curve $S^{\prime}$ in Fig. 1 this mapping would be

$$
\left(2 / \pi \zeta_{S_{S^{\prime}}} \sin u_{S^{\prime}}\right)^{1 / 2}(g J)_{S^{\prime}} \mathcal{K}_{K}^{-}\left(\zeta_{S_{S^{\prime}}}\right)=R_{S^{\prime}}+T_{S^{\prime}} \sin v
$$

A similar expression may be written along the curve $S$, but with $\left(g_{J}\right)_{S^{\prime}}, \mathfrak{K}_{K}^{-}\left(\zeta_{S^{\prime}}\right), R_{S^{\prime}}$, and $T_{S^{\prime}}$, replaced by $(g J)_{S^{\prime}}$, $\varkappa_{K}^{+}\left(\zeta_{S S^{\circ}}\right), R_{S}$ and $T_{S}$. The value of $R$ and $T$ along each of these two curves will of course be different. In determining the two values of $R$ and $T$ to be used in the evaluation of the integral in Eq. (2.19), we must take care to specify the $v$ dependence of the terms containing $\zeta, A$, and $g J$. To do this, it is convenient to use the index numbers of the stationary phase points in Fig. 1. As an example we use the determination of $R_{S^{\prime}}$ and $T_{S^{\prime}}$. Along the curve $S^{\prime}$, at the point $P_{3}$ (Fig. 1) $v$ equals $v_{3}$, $u$ equals $u_{3}$, and $\zeta_{S}$, equals $\zeta_{23}$. Thus, Eq. (2.20) yields

$$
R_{S^{\prime}}+T_{S^{\prime}} \sin v_{3}=\left(2 / \pi \zeta_{23} \sin u_{3}\right)^{1 / 2}(g J)_{3} \pi_{K}^{-}\left(\zeta_{23}\right) .
$$

Similarly, along $S^{\prime}$ at the point $P_{4} v$ equals $v_{4}$, which in tarn equals $-v_{3}, u$ equals $u_{4}$ and $\zeta_{s} s^{\prime}$ equals $\zeta_{14}$. Thus,

$$
R_{S^{\prime}}-T_{S^{\prime}}, \sin v_{3}=\left(2 / \pi_{\zeta_{14}} \sin u_{4}\right)^{1 / 2}(g J)_{4} \mathfrak{K}_{K}^{-}\left(\zeta_{14}\right) \text {. }
$$

$R_{S^{\prime}}$ and $T_{S^{\prime}}$ are evaluated from these equations. Substituting these results into Eq. (2.20), one finds that on curve $S^{\prime}$

$$
\begin{aligned}
\left(2 / \pi \zeta_{S S^{\prime}} \sin u_{S^{\prime}}\right)^{1 / 2}(g J)_{S^{\prime}}, \pi_{K}^{-}\left(\zeta_{s s^{\prime}}\right)= & \frac{1}{2}\left[\left(2 / \pi \zeta_{23} \sin u_{3}\right)^{1 / 2}(g J)_{3} \mathcal{K}_{K}^{-}\left(\zeta_{23}\right)+\left(2 / \pi \zeta_{14} \sin u_{4}\right)^{1 / 2}(g J)_{4} \mathcal{K}_{K}^{-}\left(\zeta_{14}\right)\right] \\
& +(\sin v)\left(2 \sin v_{3}\right)^{-1}\left[\left(2 / \pi \zeta_{23} \sin u_{3}\right)^{1 / 2}(g J)_{3} \mathcal{K}_{K}^{-}\left(\zeta_{23}\right)-\left(2 / \pi \zeta_{14} \sin u_{4}\right)^{1 / 2}(g J)_{4} \mathcal{K}_{K}^{-}\left(\zeta_{14}\right)\right] .
\end{aligned}
$$

Similarly on the curve $S$ one obtains

$$
\begin{aligned}
\left(2 / \pi \zeta_{S S^{\prime}} \sin u_{S^{\prime}}^{*}\right)^{1 / 2}(g J)_{S} \mathcal{K}_{K}^{+}\left(\zeta_{S S^{\prime}}\right)= & \frac{1}{2}\left[\left(2 / \pi \zeta_{23} \sin u_{3}\right)^{1 / 2}(g J)_{2} \mathcal{K}_{K}^{+}\left(\zeta_{23}\right)+\left(2 / \pi \zeta_{14} \sin u_{4}\right)^{1 / 2}(g J)_{1} \mathfrak{K}_{K}^{+}\left(\zeta_{14}\right)\right] \\
& +(\sin v)\left(2 \sin v_{2}\right)^{-1}\left[\left(2 / \pi \zeta_{23} \sin u_{3}\right)^{1 / 2}(g J)_{3} \mathfrak{K}_{K}^{+}\left(\zeta_{23}\right)-\left(2 / \pi \zeta_{14} \sin u_{4}\right)^{1 / 2}(g J)_{1} \mathfrak{K}_{K}^{+}\left(\zeta_{14}\right)\right] .
\end{aligned}
$$

We may write $f_{S^{\prime}}$ and $f_{S}$ in Eq. (2.19) in terms of $\bar{f}(u, v)$ given in Eq. (2.2). The substitution of Eqs. (2. 22a) and (2.22b) into Eq. (2.19), integration along the $v$ curves $S^{\prime}$ and $S$, and use of the definition of $d_{k}^{ \pm}\left(\zeta_{i j}\right)^{\prime}$ identical with that given for $\mathcal{d}_{\mathbb{K}}^{ \pm}\left(\zeta_{i j}\right)$ in Eq. (2.14) results in an expression for the integral,

$$
\begin{aligned}
I= & \frac{1}{4}(2 / \pi)^{1 / 2}\left\{\left[\exp \left(i A_{43}+\frac{1}{4} i \pi\right)\right]\left[\left(\zeta_{23} \sin u_{3}\right)^{-1 / 2}(g J)_{3} \mathcal{K}_{K}^{-}\left(\zeta_{23}\right) g_{k}^{-}\left(\zeta_{43}\right)+\left(\zeta_{14} \sin u_{4}\right)^{-1 / 2}(g J)_{4} \mathcal{K}_{K}^{-}\left(\zeta_{14}\right) d_{k}^{*}\left(\zeta_{43}\right)\right] .\right. \\
& \left.+\left[\exp \left(i A_{12}-\frac{1}{4} i \pi\right)\right]\left[\left(\zeta_{23} \sin u_{3}\right)^{-1 / 2}(g J)_{2} \mathfrak{K}_{K}^{+}\left(\zeta_{23}\right) \mathcal{g}_{k}^{-}\left(\zeta_{12}\right)+\left(\zeta_{14} \sin u_{4}\right)^{-1 / 2}(g J)_{1} \mathfrak{K}_{K}^{+}\left(\zeta_{14}\right) g_{k}^{+}\left(\zeta_{12}\right)\right]\right\} .
\end{aligned}
$$

As in Eqs. (26) $-(31)$ of Ref. $1(\mathrm{~h})$ it can be shown that

$$
g J=G^{-1 / 2} \bar{F}^{1 / 2} \text {, }
$$

where

$$
G=\left(\begin{array}{cc}
X_{x 0} & X_{y^{0}} \\
Y_{x^{0}} & Y_{y^{0}}
\end{array}\right), \quad \bar{F}=\left(\begin{array}{cc}
\bar{f}_{u u} & \bar{f}_{u v} \\
\bar{f}_{v u} & \bar{f}_{v v}
\end{array}\right) .
$$

Here, $X$ and $Y$ denote $f_{x}$ and $f_{y} . \quad x^{0}$ and $y^{0}$ are the values of $x$ and $y$ which characterize the initial phase in the system. From Eq. (2.6) one finds that

$$
\bar{F}=\zeta_{s s} \cdot \zeta_{Q Q^{\prime}} \sin u \sin v
$$

at any stationary phase point. Equation (2.23) may now be rewritten as ${ }^{10}$ 


$$
I=(i / 2 \pi)\left[\mathcal{K}_{K}^{-}\left(\zeta_{23}\right) \mathcal{K}_{k}^{-}\left(\zeta_{43}\right) G_{3}^{-1 / 2} e^{i f_{3}}+\mathcal{K}_{k}^{-}\left(\zeta_{14}\right) \mathfrak{K}_{K}^{+}\left(\zeta_{43}\right) G_{4}^{-1 / 2} e^{i f_{4}}+\mathcal{K}_{K}^{+}\left(\zeta_{23}\right) \mathcal{K}_{k}^{-}\left(\zeta_{12}\right) G_{2}^{-1 / 2} e^{i f_{2}}+\mathcal{K}_{K}^{+}\left(\zeta_{14}\right) \mathcal{K}_{k}^{+}\left(\zeta_{12}\right) G_{1}^{-1 / 2} e^{i f_{1}}\right]
$$

Letting the stationary phase contribution from stationary phase point $P_{j}$ be denoted by $I_{j}$, we have ${ }^{1 \mathrm{~b}}$

$$
I_{j}=(i / 2 \pi) G_{j}^{-1 / 2} \exp \left(i f_{j}\right),
$$

Eq. (2.27) can now be rearranged to yield

$$
\begin{aligned}
I= & I_{1} \mathcal{K}_{K}^{+}\left(\zeta_{14}\right) \mathcal{K}_{k}^{+}\left(\zeta_{12}\right)+I_{2} \varkappa_{K}^{+}\left(\zeta_{23}\right) \mathcal{K}_{k}^{-}\left(\zeta_{12}\right) \\
& +I_{3} \mathcal{K}_{K}^{-}\left(\zeta_{23}\right) \mathcal{K}_{k}^{-}\left(\zeta_{43}\right)+I_{4} \mathcal{K}_{K}^{-}\left(\zeta_{14}\right) \mathcal{K}_{k}^{+}\left(\zeta_{43}\right) .
\end{aligned}
$$

The results of Eq. (2.29) can also be converted to a more symmetrical form, expressed in terms of new quantities $\zeta_{21}, \ldots$ defined via the equations

$$
\left.\varkappa_{K}^{-}\left(\zeta_{12}\right) \equiv \varkappa_{K}^{+}\left(\zeta_{21}\right) \text {, etc. (definition of } \zeta_{21}\right)
$$

We then have

$$
I=\sum_{j} I_{j} \mathfrak{K}_{K}^{+}\left(\zeta_{j j_{1}}\right) \mathfrak{K}_{k}^{+}\left(\zeta_{j j_{2}}\right)
$$

$j_{1}$ and $j_{2}$ are the nearest neighbors of $j$. The generalization of $r$-dimensions is

$$
I=\sum_{j} I_{j} \varkappa_{K_{1}}^{+}\left(\zeta_{j j_{1}}\right) \ldots \boldsymbol{\kappa}_{K_{r}}^{+}\left(\zeta_{j j_{r}}\right)
$$

where $K_{1} \ldots K_{r}$ are the integers appearing in the original expression for $I\left[K_{1}=K, K_{2}=k\right.$ in (2.31)]. The prescription for using Eq. (2.32) is as follows; whenever some $f_{i}-f_{j}$ is positive, that is, disagrees with Eq. (2.6), the corresponding $\mathfrak{K}_{K_{r}}^{+}\left(\zeta_{i j}\right)$ term is replaced by $\mathfrak{K}_{K_{r}}^{-}\left(\zeta_{j i}\right)$.

The Bessel result in Ref. 1(i) was shown to include the Airy result of Ref. $1(\mathrm{c})$ of this series as a limiting case. Similarly, the Bessel result in Eq. (2.32) can be shown to include the Airy result, Eq. (39), of Ref. $1(\mathrm{~h})$ as a limiting case. ${ }^{11}$

Returning to the case $r=2$, inspection of Eq. (2.29) [keeping Eq. (2.19) in mind] shows that as all $\zeta^{\prime} s$ become large the integral goes over to the sum of stationary phase contributions,

$$
I=\sum_{i=1}^{4} I_{i}
$$

In some physical problems we expect that only a particular pair of $\zeta$ 's will become large. Then from Fig. 1 if the curves $S^{\prime}$ and $S$ are far apart we expect from Eq. (2. 9), $\zeta_{14}$ and $\zeta_{23}$ to become large. If this occurs, Eq. (2. 29) becomes

$$
I=I_{1} \mathcal{K}_{k}^{+}\left(\zeta_{12}\right)+I_{2} \mathcal{K}_{k}^{-}\left(\zeta_{12}\right)+I_{3} \mathfrak{K}_{k}^{-}\left(\zeta_{43}\right)+I_{4} \mathfrak{K}_{k}^{+}\left(\zeta_{43}\right) .
$$

We shall call this result the Bessel uniform-in-pairs approximation. A similar situation arises if instead the curves $Q^{\prime}$ and of $Q$ are far apart. Then $\zeta_{12}$ and $\zeta_{43}$ would become large and the resulting equation would be of the same form as Eq. (2, 34).

\section{UNIFORM APPROXIMATION FOR REAL PLUS COMPLEX STATIONARY PHASE POINTS}

Although the completely uniform approximation, Eq. (2. 29), was developed for the case of four real station- ary phase points, a similar treatment can be used for some instances involving four complex valued stationary phase points. However, this completely uniform result cannot be used for the case of two real and two complex stationary phase points. The problem lies in the fact that the assumed model as stated in Eqs. (2.1) and (2.2) does not allow the construction of $u$ curves or $v$ curves along which one extremum is at real values of $(u, v)$ while a second is at a complex value of $(u, v)$. Instead, we treat this situation in the following more restricted way.

We shall consider the case where the complex and real points are well separated and apply the Bessel uniformin-pairs Eq. (2.34) in complex form. Complex roots of Eqs. (2. 3a) and (2.3b) occur if $\zeta<k$. One solution of $(2,3 a)$ would be the purely imaginary value $v_{Q^{\prime}}=i \bar{v}_{Q}$. However, $v_{Q^{\prime}}=2 n \pi+i \bar{v}_{Q^{\prime}}$ where $n$ is any positive or negative integer is also a solution. As $\zeta$ approaches $k$ from above (i.e., from the region $\zeta>k$ ), $\bar{v}_{Q^{\prime}}$ tends to zero. Thus, of the roots for $v_{Q}$, in the region $\zeta<k$, only the one with $n=0$ applies, by analytical continuation from the domain of real roots $v_{Q^{\prime}}$. A similar conclusion applies to $v_{Q}$. That is, the real part of the complex roots is to be set equal to zero,

$$
\operatorname{Re}\left(v_{Q}\right)=\operatorname{Re}\left(v_{Q}\right)=0 .
$$

The equations for $A_{Q Q^{\prime}}$ and $v_{Q}$, corresponding to Eqs. (2.6)-(2.8), are found to be

$$
\begin{aligned}
& A_{Q Q^{\prime}}=\frac{1}{2}\left(f_{Q^{\prime}}+f_{Q}\right), \\
& i k\left(v_{Q^{\prime}}^{\prime \prime}-\tanh v_{Q^{\prime}}^{\prime \prime}\right)=\frac{1}{2}\left(f_{Q^{\prime}}-f_{Q}\right),
\end{aligned}
$$

where

$$
v_{Q^{\prime}}^{\prime \prime}=\operatorname{Im}\left(v_{Q^{\prime}}\right) \text {; }
$$

$\zeta$ is given by

$$
\zeta=k / \cosh v_{Q^{\prime}}^{\prime \prime} \text {. }
$$

In this case of complex roots $f_{Q^{\prime}}$ equals to complex conjugate of $f_{Q}, f_{Q}^{*}$, and causes the rhs of Eq. (3.2) to be imaginary. Writing Eq. (3.3) in a different form

$$
i k \cosh ^{-1}(k / \zeta)-i\left(k^{2}-\zeta^{2}\right)^{1 / 2}=\frac{1}{2}\left(f_{Q^{\prime}}-f_{Q}\right),
$$

we can see that the rhs of Eq. (3.5) has as its range the positive imaginary axis. Therefore,

$$
\operatorname{Im}\left(f_{Q^{\prime}}\right)>0 \text { and } \operatorname{Im}\left(f_{Q}\right)<0 \text {. }
$$

\section{SYMMETRY-INCREASED NUMBER OF STATIONARY PHASE POINTS}

If, because of symmetry, there are more than four stationary phase points in the $(2 \pi)^{2}$ area of integration in Eq. (1.1), the above formalism can be extended to cover this case. As a simple example we consider first the one-dimensional integral

$$
I=\int_{-\pi}^{\pi} g(x) \exp [i f(x)] d x
$$

When the following periodicity conditions hold 


$$
\begin{aligned}
& f(x+2 \pi / N)=f(x)+2 \pi M, \\
& g(x+2 \pi / N)=g(x),
\end{aligned}
$$

where $N$ and $M$ are integers, Eq. (3.1) becomes

$$
I=N \int_{-\pi / N}^{\pi / N} g(x) \exp [i f(x)] d x .
$$

If one next maps $f(x)$ onto a function of a new variable, $\bar{u}$, in a $2 \pi / N$ interval,

$f(x) \equiv \bar{f}(\bar{u})=\bar{K} \bar{u}-\zeta \sin (N \bar{u})+A, \quad-\frac{\pi}{N}<x<\frac{\pi}{N}, \quad-\frac{\pi}{N}<\bar{u}<\frac{\pi}{N}$

and makes the additional change of variable $u=N \bar{u}$, one obtains

$$
\left.I=N \int_{-\pi}^{\pi} \bar{g}(u) \bar{J} \exp i \frac{\bar{K}}{N} u-\zeta \sin u+A\right) d u
$$

where $\vec{g}(u)$ equals $g(x)$ and where $\vec{J}$ is the Jacobian of the transformation from $x$ to $u$. The uniform approximation method is then applied to Eq. (4.6). Thus, to use the Bessel uniform approximation method for this case, the $K$ in Eqs. (2.1)-(2.29) is replaced, one sees from Eq. (4.6), by $\bar{K} / N$. This latter number is still an integer in the problems considered in the next paper, because selection rules cause $K$ to be a multiple of $N$. Similar remarks apply to the multidimensional integral in Eqs. (1.1) and (1.2).

*Supported in part by a grant from the National Science Foundation.

${ }_{1}^{1}$ (a) R. A. Marcus, Chem. Phys. Lett. 7, 252 (1970); (b) R. A. Marcus, J. Chem. Phys. 54, 3965 (1971); (c) J. N. L. Connor and R. A. Marcus, ibid. 55, 5636 (1971); (d) W. H. Wong and R. A. Marcus, ibid. 55, 5663 (1971); (e) J. Stine and R. A. Marcus, Chem. Phys. Lett. 15, 536 (1972); (f) R. A. Marcus, J. Chem. Phys. 56, 311 (1972); (g) 56, 3548 (1972); (h) 57, 4903 (1972); (i) J. Stine and R. A. Marcus, ibid. 59, 5145 (1973); (j) R. A. Marcus, ibid. 59, 5135 (1973); (k) Discuss. Faraday Soc. 55, 34 (1973). (1) D. E. Fitz and R. A. Marcus, J. Chem. Phys. 59, 4380 (1973). (m) H. Kreek and R. A. Marcus, J. Chem. Phys. 61, 3308 (1974). ${ }^{2}$ (a) W. H. Miller, J. Chem. Phys. 53, 1949 (1970); (b) 53, 3578 (1970); (c) 54, 5386 (1971); (d) Chem. Phys. Lett. 7, 431 (1970); (e) W. H. Miller and T. F. George, J. Chem. Phys. 56, 5668 (1972); (f) 57, 2458 (1972); (g) C. C. Rankin and W. H. Miller, ibid. 55, 3150 (1971); (h) J. D. Doll and W. H. Miller, ibid. 57, 5019 (1972); (i) J. D. Doll, T. F. George, and W. H. Miller, ibid. 58, 1343 (1973); (j) W. H. Miller and A. W. Rackowski, Discuss. Faraday Soc. 55, 45 (1973).

${ }^{3}$ J. N. L. Connor, Mol. Phys. 25, 181 (1973); J. N. L. Connor, Discuss. Faraday Soc. 55, 51 (1973).

${ }^{4}$ Other examples of recent semiclassical work include: (a) $R$. D. Levine and B. R. Johnson, Chem. Phys. Lett. 7, 404 (1970); (b) P. Pechukas, Phys. Rev. 181, 166, 174 (1969); (c) I. I. Beigman, L. A. Varnshtein, and I. I. Sobel'man, Zh. Eksp. Teor. Fiz. 57, 1703 (1969); [Sov. Phys. JETP 30, $920(1970)$ ]; (d) I. C. Percival and D. Richards, J. Phys. B 3, 315, $1035(1970)$. (e) J. M. Bowman and Aron Kuppermann, Chem. Phys. Lett. 19, 166 (1973).

${ }^{5}$ S. F. Wu and R. D. Levine, Mol. Phys. 25, 937 (1973); J. J. Tyson, R. P. Saxon and J. C. Light, J. Chem. Phys. 59, 363 (1973); and R. A. Labudde, Chem. Phys. Lett. 13, 154 (1972).

${ }^{6}$ The mapping in (2.1) and later in (2.9) yields the same final result as that in Ref. 1(i). We are indebted to Mr. Stine for a helpful discussion of this and of related points.

${ }^{7}$ These curves are not necessarily curves on which $v$ is a constant. (i.e., they are not necessarily $u$-coordinate curves.)

${ }^{8} \mathrm{H}$. Hancock, Theory of Maxima (Dover, New York, 1960), pp. $23 \mathrm{ff}$.

${ }^{9}$ M. Abramowitz and I. A. Stegun, U. S. Natl. Bur. Stand. Appl. Math, Ser. 55, 355 (1964).

${ }^{10}$ We use the relations, deduced from $(2.3):\left(\sin u_{2}\right)^{1 / 2}$ $=i\left(\sin u_{3}\right)^{1 / 2} ;\left(\sin u_{1}\right)^{1 / 2}=i\left(\sin u_{4}\right)^{1 / 2} ;\left(\sin v_{1}\right)^{1 / 2}=i\left(\sin v_{2}\right)^{1 / 2}$; $\left(\sin v_{4}\right)^{1 / 2}=i\left(\sin v_{3}\right)^{1 / 2}$.

${ }^{11} \mathrm{~J}$. N. L. Connor (private communication; unpublished): M. Abramowitz and I. A. Stegun (Eds.), Ref. 9, p. 366. We are indebted to Dr. Connor for calling this reference to our attention. 